\title{
Identification and characterization of a laminin-binding protein of Aspergillus fumigatus: extracellular thaumatin domain protein (AfCalAp)
}

\author{
Santosh Kumar Upadhyay, ${ }^{1,2}$ Lakshna Mahajan, ${ }^{1}$ Sandhya Ramjee, ${ }^{3}$ \\ Yogendra Singh, ${ }^{1}$ Seemi Farhat Basir ${ }^{2}$ and Taruna Madan ${ }^{1,3}$ \\ ${ }^{1}$ Institute of Genomics and Integrative Biology, Mall Road, Delhi 110007, India \\ ${ }^{2}$ Department of Biosciences, Jamia Millia Islamia, New Delhi 110025, India \\ ${ }^{3}$ National Institute for Research in Reproductive Health (NIRRH), Parel, Mumbai 400012, India
}

Correspondence

Taruna Madan

taruna_m@hotmail.com

Received 20 August 2008

Accepted 10 February 2009

\begin{abstract}
Aspergillus fumigatus, an opportunistic fungal pathogen, infects the human host via inhalation of airborne conidia. Adhesion of fungal conidia, to host cells and extracellular matrix (ECM) components associated with host tissue surfaces, is thought to be the primary step in the pathogenesis and dissemination of infection. To identify novel adhesion proteins (adhesins) of $A$. fumigatus, we screened its proteome in silico using SPAAN (software program for prediction of adhesins and adhesin-like proteins using neural networks). One of the predicted adhesinencoding genes with a $P_{\text {ad }}$ (probability of being adhesin) value $>0.9$, the gene encoding extracellular thaumatin domain protein (AfCalA), was cloned and expressed in Escherichia coli. Recombinant AfCalAp showed significant binding with laminin and murine lung cells. AntiAfCalAp antibodies inhibited the binding of AfCalAp to laminin in a dose-dependent manner. Significant binding of anti-AfCalAp antibodies to $2 \mathrm{~h}$ swollen conidia suggests the presence of AfCalAp on the conidial surface. AfCalA transcript was not detectable in resting conidia but was detected in conidia incubated with RPMI 1640 medium in the presence and absence of lung epithelial cell line (A539)-derived ECM. Elevated levels of lgE antibodies specific to AfCalAp were observed in the sera of two out of seven patients with allergic bronchopulmonary aspergillosis. The study confirms the relevance of the bioinformatic approach for predicting fungal adhesins and establishes AfCalAp as a novel laminin-binding protein of $A$. fumigatus.
\end{abstract}

\section{INTRODUCTION}

Aspergillus fumigatus, an opportunistic lung pathogen, is the main aetiological agent of human aspergillosis. The increasing incidence of both allergic aspergillosis and fatal invasive aspergillosis, and the limited success of currently available therapy, has prompted the search for novel therapeutic strategies. Adhesion of inhaled airborne $A$. fumigatus conidia to the host lung epithelial cells and extracellular matrix (ECM) components is thought to be one of the primary steps for initiation of infection (Annaix et al., 1992). Lungs with damaged epithelial lining and exposed ECM are more susceptible to infection by $A$. fumigatus (Bodey \& Vartivarian, 1989; Coulot et al., 1994).

Abbreviations: ABPA, allergic bronchopulmonary aspergillosis; DAPI, 4',6-diamidino-2'-phenylindole dihydrochloride; ECM, extracellular matrix; FITC, fluorescein isothiocyanate; GPI, glycosylphosphatidylinositol; GST, glutathione $S$-transferase; $P_{\text {ad, }}$, probability of being adhesin.

Figures showing protein sequence alignments for AfCalAp and expression analysis of the RNA are available as supplementary data with the online version of this paper.
ECM proteins of the host, namely fibronectin, laminin, collagens and proteoglycans, are implicated in attachment of a number of fungal pathogens (Gaur \& Klotz, 1997; Gil et al., 1996; Gonzalez et al., 2005; Lopez-Ribot \& Chaffin, 1994; Mendes-Giannini et al., 2006; Tronchin et al., 1997; Wasylnka \& Moore, 2000). Adhesion of A. fumigatus conidia to fibrinogen is mediated by the $\mathrm{D}$ domain of fibrinogen (Annaix et al., 1992), and increases with the maturation of conidia (Coulot et al., 1994).

Adherence is indicative that the fungal factors recognize ligands (protein or carbohydrate) on the surface of host cells or constituent(s) of the ECM. These pathogen factors, with an affinity towards ECM component(s), are envisaged to contribute to the virulence. Several studies have implicated cell wall proteins or carbohydrates of $A$. fumigatus as being adhesins, and characterized their interactions with ECM components (Annaix et al., 1992; Bouchara et al., 1997; Penalver et al., 1996; Thau et al., 1994). Sturtevant \& Latge (1992) identified a $54-58 \mathrm{kDa}$ conidial surface protein that binds human complement component C3 and/or a C3 fragment. Two polypeptides, 
with molecular masses of 23 and $30 \mathrm{kDa}$, present in whole conidial homogenate and 2-mercaptoethanol extract from isolated conidial cell walls, respectively, are reported to specifically interact with fibronectin (Penalver et al., 1996). Interaction of $A$. fumigatus conidia with laminin has been shown to be mediated by a $72 \mathrm{kDa}$ laminin-binding protein expressed at the cell wall surface of resting conidia (Tronchin et al., 1997). Gil et al. (1996) identified one polypeptide with an apparent molecular mass of $37 \mathrm{kDa}$ from whole conidial homogenates that specifically interacts with laminin. The rodletless mutant of A. fumigatus displayed reduced collagen affinity, but retained affinity with pneumocytes, fibrinogen and laminin (Thau et al., 1994).

In view of the multifactorial nature of conidial adhesion, the relevance of the adhesins for understanding pathogenesis and the plausible application of anti-adhesins for the prevention and therapy of aspergillosis, we attempted global screening of the A. fumigatus proteome to identify putative adhesins using a bioinformatics-based approach.

\section{METHODS}

\section{Bioinformatic analysis}

Prediction of adhesion proteins of $\boldsymbol{A}$. fumigatus using an in silico approach. SPAAN, a software program for amino acid sequence based prediction of adhesins and adhesin-like proteins, was used for the prediction of potential adhesins in the A. fumigatus proteome. Sequences of A. fumigatus proteins were downloaded from the website of the Institute of Genomic Research (http://www.tigr.org/tdb/e2k1/afu1/).

Amino acid sequence analysis of AfCalAp. PSORT II (http:// psort.nibb.ac.jp/form2.html) and SignalP 3.0 (http://www.cbs.dtu. $\mathrm{dk} /$ services/SignalP/) were used to determine the cellular localization and to identify the signal peptide of AfCalAp, respectively. The thaumatin domain was identified using InterProScan software (http:// www.ebi.ac.uk/Tools/InterProScan/). Sequence alignment of AfCalAp with two previously known thaumatin consensus containing proteins was carried out using Muscle software (http://www.ebi.ac.uk/Tools/ muscle/index.html).

A. fumigatus strain. A. fumigatus strain Af293, isolated from the lungs of an invasive aspergillosis patient, was kindly provided by Dr D. W. Denning (School of Medicine, University of Manchester, Manchester, UK). This is the same strain that was used in the past for the whole genome sequencing project by the Sanger Institute (Hinxton, Cambridge, UK) and the Institute of Genomic Research (TIGR, Rockville, MD, USA).

Isolation of $\boldsymbol{A}$. fumigatus conidia. A. fumigatus was freshly subcultured on Sabouraud dextrose agar (Himedia). Conidia were harvested after $72 \mathrm{~h}$ growth, using PBS and were pelleted by centrifugation at $12000 \mathrm{~g}$ for $10 \mathrm{~min}$ at $4{ }^{\circ} \mathrm{C}$. The conidial pellet was washed twice with PBS and stored in fresh PBS at $4{ }^{\circ} \mathrm{C}$ until use. Before use in experiments, the pellet was resuspended in fresh PBS and an aliquot of the conidia (i.e. resting conidia) was further diluted and quantified using a haemocytometer to count the number of colony forming units.

Incubation of conidia with lung epithelial cell line (A549)derived ECM. The A549 matrix was generated as described by
Bromley \& Donaldson (1996) with some modifications. Monolayers of A549 lung epithelial cell line (procured from the National Centre for Cell Science, Pune, India) were prepared in the cell culture flasks. To remove cells from the matrix, the confluent cell layer was washed with sterile PBS and incubated with $0.1 \%$ sodium azide for $90 \mathrm{~min}$. This treatment was followed by an incubation with $4 \%$ sodium deoxycholate and $0.1 \%$ sodium azide for $1 \mathrm{~h}$ at room temperature. The flasks were washed twice with PBS, $0.05 \%$ Tween 20 , followed by a wash with PBS, and the surface was microscopically examined to ensure removal of the cells. The presence of ECM on the flask surface was confirmed using Bradford reagent (Sigma) for protein estimation (Bradford, 1976).

A total of $2 \times 10^{9}$ A. fumigatus conidia were suspended in $30 \mathrm{ml} \mathrm{RPMI}$ 1640 medium (Sigma) and incubated in the culture flask containing the A549-derived ECM ( $1 \mathrm{~h}$ swollen conidia with ECM), whereas, in the control flask, conidia were incubated with RPMI 1640 medium ( $1 \mathrm{~h}$ swollen conidia) at $37^{\circ} \mathrm{C}$ for $1 \mathrm{~h}$. The medium was then aspirated and replaced with ice-cold Dulbecco's PBS. The conidia adhered to the culture flasks were dislodged using a cell scraper and were pelleted at $14000 \mathrm{~g}$ for $5 \mathrm{~min}$ at $4{ }^{\circ} \mathrm{C}$. These conidial pellets were quickly frozen using liquid nitrogen and used for RNA isolation. For examining the conidial surface reactivity of anti-AfCalAp serum, conidia obtained after $2 \mathrm{~h}$ incubation with RPMI 1640 ( $2 \mathrm{~h}$ swollen conidia) were suspended in Dulbecco's PBS and subjected to a further step (endogenous peroxidase inactivation).

Isolation of total RNA from conidia. Frozen conidial pellets were ground to a fine powder in the presence of liquid nitrogen using a mortar and pestle. Total RNA was isolated using an EZ-RNA isolation kit (Biological Industries), DNase treated and the RNA cleaned using the RNeasy mini protocol (Qiagen). The quality of total RNA was confirmed by assessing the $A_{260} / A_{280}$ ratio and looking for prominent intact $26 \mathrm{~S}$ and $18 \mathrm{~S}$ ribosomal bands without any genomic DNA contamination by gel electrophoresis.

RT-PCR. First strand cDNA synthesis from the fungal RNAs was achieved using M-MuLV reverse transcriptase (New England Biolabs) as per the manufacturer's protocol. The enzyme was inactivated at $90{ }^{\circ} \mathrm{C}$ for $10 \mathrm{~min}$. These cDNAs were used as templates in PCRs using gene-specific primers designed with the help of PrimerSelect software (DNASTAR). AfCalA-specific forward and reverse primers contained BamHI and Xho restriction endonuclease sites, respectively, as underlined in the following primer sequences: $\mathrm{Fp}=5^{\prime}$-GGATCCACATGATGTTCACCAAG- $3^{\prime}$ and $\mathrm{Rp}=5^{\prime}$-CTCGAGCTA $\overline{\text { GTTGCC- }}$ AATGTTC-3'. Primers for Actin were designed to target an exonexon boundary so as to give multiple amplification products in case of any genomic DNA contamination in cDNA (data not shown). The sequences of these primers were as follows: Fp $=5^{\prime}$-AACTTCCCGATGGTCAGGTCA-3', Rp =5' -AGAGGCCAGAATGGAACCAC-3' .

Cloning, expression and purification of AfCalAp. The $\mathrm{DH} 5 \alpha$ strain of Escherichia coli was used for cloning. Gel-purified PCR product was cloned in pGEMT-easy vector (Promega) using the manufacturer's protocol. The insert was cleaved out of the pGEMTeasy vector by $\mathrm{BamHI} / \mathrm{XhoI}$ digestion and was cloned into pGEX$5 \mathrm{X}-3$ vector between the Bam HI and XhoI sites. Cloning and gene identity were confirmed by restriction digestion and by sequencing of the cloned insert, respectively. For expression, the plasmid was used to transform the BL-21 strain of E. coli. Recombinant protein expression was induced with the addition of $0.2 \mathrm{mM}$ IPTG to the exponential phase bacterial culture $\left(\mathrm{OD}_{600}=0.6\right)$ and growth for $4 \mathrm{~h}$ at $30{ }^{\circ} \mathrm{C}$ in Luria broth. The recombinant protein was purified by glutathione affinity chromatography as described previously (Saxena et al., 2003). Expressed glutathione S-transferase (GST) fusion protein, as visualized using SDS-PAGE, showed an approximate molecular mass of $45 \mathrm{kDa}$ (Fig. 1). The yield of the protein was 


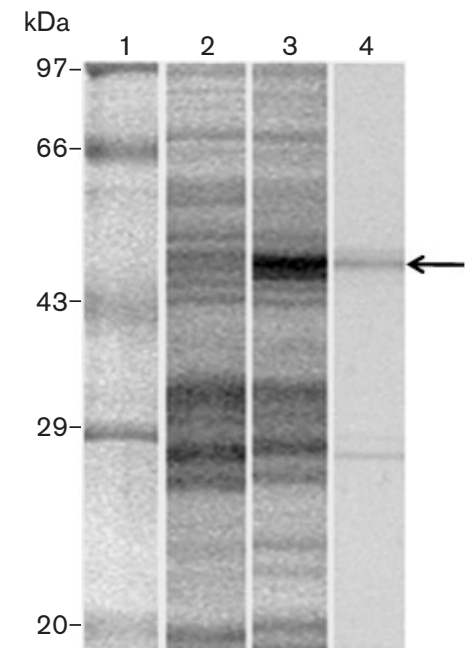

Fig. 1. SDS-PAGE (12\% gel) showing the expression and purification of the AfCalAp-GST fusion protein (size $=45 \mathrm{kDa}$ approx.). Coomassie brilliant blue staining was used to visualize the electrophoresed proteins on the gel. Lane 1, protein molecular mass marker; lane 2, bacterial (BL21) lysate of uninduced cells containing AfCalAp-pGEX clone; lane 3, bacterial (BL21) lysate of (0.2 mM) IPTG-induced cells containing AfCalAp-pGEX clone; lane 4, affinity purified AfCalAp-GST fusion protein.

approximately $2 \mathrm{mg}$ ( 1 induced bacterial culture $)^{-1}$. In view of the low yield of protein, induction of the bacterial culture was done at a large scale to get a sufficient amount of recombinant protein for further studies. Purified protein was dialysed to remove DTT and glutathione using dialysis membrane with a $12 \mathrm{kDa}$ cut-off (Sigma). The GST-tagged fusion protein was subjected to factor Xa (Qiagen) cleavage using the manufacturer's protocol, followed by SDS-PAGE. The AfCalAp band was excised from the gel and the purified recombinant protein was obtained by electroelution for use in immunological characterization and production of anti-AfCalAp serum. Recombinant L3 ribosomal protein of A. fumigatus, used as a control during the study, was expressed and purified as described previously (Saxena et al., 2003).

Raising polyclonal anti-AfCalAp in mice. With an approved protocol from the Institute of Genomics and Integrative Biology animal ethics committee, specific-pathogen-free 4- to 6-week-old $\mathrm{BALB} / \mathrm{c}$ mice were obtained from the animal house facility of the National Institute of Immunology (New Delhi, India), and were intraperitoneally immunized with $25 \mu \mathrm{g}$ recombinant AfCalAp in $100 \mu \mathrm{l}$ PBS (pH 7.4) and $100 \mu$ Freund's complete adjuvant (Sigma). At 2 and 4 weeks following the primary immunization, intraperitoneal booster immunizations were performed with $25 \mu \mathrm{g}$ protein in Freund's incomplete adjuvant (Sigma). Control mice were immunized with the adjuvant and PBS. All the mice were sacrificed 2 weeks after the second booster immunization and sera collected. The titre of the anti-AfCalAp serum was evaluated by its immunoreactivity with recombinant AfCalAp on the dot-blots.

\section{Expression of AfCalAp on the conidial surface}

ELISA. The $2 \mathrm{~h}$ swollen conidia $\left(2 \times 10^{8}\right.$ per well in a 96-well culture plate) were incubated with Dulbecco's PBS containing $1 \%(\mathrm{v} / \mathrm{v})$ $\mathrm{H}_{2} \mathrm{O}_{2}$ for 15 min to block the endogenous peroxidase reactivity. After washing, the conidia were incubated overnight at $4{ }^{\circ} \mathrm{C}$ in Dulbecco's
PBS (Himedia). Unbound conidia were removed by washing twice with Dulbecco's PBS. Control or anti-AfCalAp mouse sera were diluted 1:50 in Dulbecco's PBS containing 1.5\% BSA and were added to separate wells. After incubation at $37{ }^{\circ} \mathrm{C}$ for $1 \mathrm{~h}$, the wells were washed twice with Dulbecco's PBS and incubated for $2 \mathrm{~h}$ with protein A-horseradish peroxidase conjugate (Bangalore Genei) diluted 1:750 in Dulbecco's PBS containing 1.5\% BSA. After washing once with PBS, $0.05 \%$ Tween 20, and twice with PBS, AfCalAp expression on conidia was detected using chromogen ortho-phenyldiamine and $\mathrm{H}_{2} \mathrm{O}_{2}$ as substrate. The reaction was stopped using $4 \mathrm{M} \mathrm{H}_{2} \mathrm{SO}_{4}$ and the absorbance was read at $492 \mathrm{~nm}$.

Immunofluorescence (laser scanning) microscopy. The 2 h swollen conidia ( $10^{7}$ cells) were fixed in $100 \mu \mathrm{l} 4 \%$ paraformaldehyde in PBS at room temperature for $20 \mathrm{~min}$. They were smeared on poly-L-lysine (Sigma) coated clean glass slides and left to air dry for $10 \mathrm{~min}$. Nonspecific interactions were blocked using $5 \%$ normal goat serum (blocking solution) (Bangalore Genei) for $45 \mathrm{~min}$ at room temperature in a moist chamber. Slides were incubated overnight with mice sera (anti-AfCalAp or control) at 1:50 dilution in blocking solution at $4{ }^{\circ} \mathrm{C}$. After three washes with PBS, the smears were incubated with phycoerythrin-labelled anti-mouse IgG raised in goat (Sigma) for $1 \mathrm{~h}$ at room temperature in moist conditions. After three washes, the slides were counterstained with DAPI (4',6-diamidino-2' phenylindole dihydrochloride) (Roche Diagnostics) and mounted in Vectashield (Vector Laboratories). Serial sections of the fluorescent cells were obtained with a Zeiss LSM 510 meta laser scanning confocal microscope, using a 488 argon ion laser (Carl Zeiss). The gain settings etc. were optimized for each image. Serial sections ( $x y$ plane) were obtained at $0.5 \mu \mathrm{m}$ intervals for conidia. Three-dimensional reconstructions were obtained by the resident software.

\section{Interaction of AfCalAp with murine lung and spleen cell suspension}

Preparation of murine lung and spleen cell suspension. Lung and spleen tissues from naive BALB/c mice were minced in the presence of ice-cold Dulbecco's PBS (Himedia) between slides with a frosted surface. A sterile nylon mesh was used to remove the tissue debris. Red blood cells were removed by incubating the cell suspension with red blood cell lysis buffer $\left(1.04 \mathrm{~g} \mathrm{NH}_{4} \mathrm{Cl}, 0.125 \mathrm{~g} \mathrm{NaHCO}_{3}\right.$ and $0.01 \mathrm{~g}$ EDTA in $50 \mathrm{ml}$ MilliQ water) for $5 \mathrm{~min}$ at room temperature. After two washes in Dulbecco's PBS, the cells were suspended in ice-cold FACS buffer $(0.5 \%$ BSA, $0.1 \%$ sodium azide in Dulbecco's PBS). Microscopic examination confirmed that these were single cell suspensions. The number of cells was counted on a haemocytometer.

Fluorescein isothiocyanate (FITC)-labelling of AfCalAp-GST and GST. Recombinant AfCalAp-GST and GST were labelled with FITC (Sigma) using a protocol described by Holmes \& Fowlkes (1996).

Flow cytometry. Lung or spleen cell suspensions $\left(300 \mu \mathrm{l} 7.5 \times 10^{5}\right.$ cells $\mathrm{ml}^{-1}$ in FACS buffer) were incubated with $9 \mu \mathrm{g}$ FITC-labelled AfCalAp or equivalent moles of FITC-labelled GST for $45 \mathrm{~min}$ at $4{ }^{\circ} \mathrm{C}$. After three washes with FACS buffer, the cells were fixed in $4 \%$ formaldehyde in PBS. Five-thousand cells from each sample were assayed using an EasyCyte plus flow cytometry system (Guava Technologies). WinMDI version 2.8 was used for analysis of flowcytometry data. The number of cells with associated FITC fluorescence was used as a measure of the labelled AfCalAp protein binding to lung/spleen cells.

Interaction of AfCalAp with various ECM components. A total of $1.5 \mu \mathrm{g}$ each ECM component [all from Sigma: collagen type I from rat tail, decorin from bovine articular cartilage, fibrinogen from bovine plasma, fibronectin from human plasma, laminin from EngelbrethHolm-Swarm murine sarcoma (basement membrane)] or control 
protein (BSA; Sigma) was spotted on nitrocellulose membranes. After blocking with $3 \%$ skimmed milk powder, the dot-blot membranes were incubated with AfCalAp-GST ( $40 \mu \mathrm{g}$ protein diluted in $2.5 \mathrm{ml}$ tris-buffered saline, $0.05 \%$ Tween 20 (TBST), containing $3 \%$ skimmed milk powder) or equal amounts of GST on a shaker for $3 \mathrm{~h}$ at $37^{\circ} \mathrm{C}$. After two TBST washes, the membranes were incubated with anti-GST peroxidase (Promega; 1:2000 diluted in TBST containing $3 \%$ skimmed milk powder) for $3 \mathrm{~h}$ at $37^{\circ} \mathrm{C}$. Membranes were washed twice with and once with tris-buffered saline (TBS) and the reaction was detected by $3,3^{\prime}$ diaminobenzidine and hydrogen peroxide.

Interaction of AfCalAp with laminin. A total of $2 \mu \mathrm{g}$ per well (Maxisorp; Nunc) of laminin in PBS $\left(0.02 \mu \mathrm{g} \mu \mathrm{l}^{-1}\right)$ was incubated overnight at $4{ }^{\circ} \mathrm{C}$. After blocking the samples in the wells with $3 \%$ skimmed milk powder, $1 \mu \mathrm{g}$ each of test (AfCalAp) or control (L3 ribosomal protein) proteins were added to different wells and incubated for $2 \mathrm{~h}$ at $37^{\circ} \mathrm{C}$. In the binding inhibition experiments, AfCalAp was mixed with various dilutions of anti-AfCalAp serum before its addition to different wells. Anti-GST peroxidase (Promega; 1:2000 diluted in PBS containing 3\% skimmed milk powder) was added and binding of AfCalAp was probed using chromogen orthophenyldiamine and $\mathrm{H}_{2} \mathrm{O}_{2}$.

Human sera. Following the guidelines of the Institute of Genomics and Integrative Biology ethics committee, the patients' sera $(n=7)$ used in the study were obtained from clinically confirmed cases of allergic bronchopulmonary aspergillosis (ABPA) [satisfying Rosenberg's criteria (Rosenberg et al., 1977)] registered at the V. Patel Chest Institute, Delhi, India. Sera obtained from healthy, consenting donors $(n=7)$ with no history of pulmonary disease were used as controls.

\section{IgG and IgE antibodies specific to AfCalAp in the sera of ABPA} patients. A total of $2 \mu \mathrm{l}$ electroeluted GST-tag less AfCalAp ( $1 \mu \mathrm{g}$ $\mu^{-1}$ ) was spotted onto nitrocellulose membrane. To determine the IgG and IgE reactivity of AfCalAp, the dot-blots containing AfCalAp spots were incubated with patient/control serum (1:100 diluted in TBST for IgG reactivity and $1: 20$ diluted in TBST for IgE reactivity). Binding of $\operatorname{IgG}$ and IgE antibodies was probed using protein Ahorseradish peroxidase conjugate (1:750 diluted in TBST containing $3 \%$ skimmed milk powder) and anti-IgE-horseradish peroxidase conjugate (1:750 diluted in TBST containing 3\% skimmed milk powder), respectively. Membranes were washed and the reaction was detected as described above.

\section{RESULTS AND DISCUSSION}

The current study presents a bioinformatics-based approach for the identification of adhesion proteins in $A$. fumigatus and other fungi, and validates the approach by characterizing the adhesin nature of recombinant extracellular thaumatin domain protein (AfCalAp) of $A$. fumigatus, predicted as a potential adhesin by SPAAN software.

\section{Bioinformatic analysis of AfCalAp}

To identify novel adhesion proteins (adhesins) of $A$. fumigatus, we screened its proteome in silico using SPAAN. This software was developed by employing 105 compositional properties and artificial neural networks, and has been validated for the prediction of bacterial adhesins (Sachdeva et al., 2005). SPAAN had an optimal sensitivity of
$89 \%$ and specificity of $100 \%$ on a defined test set, and could identify $97.4 \%$ of known adhesins at a high $P_{\text {ad }}$ (probability of being adhesin) value from a wide range of bacteria. As the adhesins used for training this program belonged to microbes from diverse classes of organisms and one third (approx.) of non-adhesins employed for this purpose were from Saccharomyces cerevisiae, we envisaged SPAAN to be useful for the prediction of fungal adhesins. Among 9894 proteins screened, $0.83 \%(n=82)$ had a $P_{\text {ad }}$ value $>0.9$ ( 25 of them are listed in Table 1), $2.47 \%$ $(n=243)$ proteins had a $P_{\text {ad }}$ value $>0.8$, whereas, those having a $P_{\text {ad }}$ value $>0.5$ constituted $11.62 \%(n=1149)$ of the proteome. The mean $P_{\text {ad }}$ value per protein was 0.274 . RodA hydrophobin, known to have a role in the adhesion of $A$. fumigatus, was assigned with a $P_{\text {ad }}$ value of 0.79 . In view of its good adhesion potential $\left(P_{\mathrm{ad}}>0.9\right)$, its small ORF size (534 bp) facilitating cloning/expression and an earlier study describing its presence on the fungal cell wall (Belaish et al., 2008), we selected AfCalAp (Afu3g09690) for further in vitro studies. Serine, threonine and proline were found to constitute $24.89 \%$ of the total amino acids in the sequence of AfCalAp, i.e. a characteristic typical of adhesins (Levdansky et al., 2007). SPAAN assigned a $P_{\text {ad }}$ value of 0.942171 to AfCalAp, whereas, L3 ribosomal protein (Afu2g11850) was assigned a comparatively low $P_{\mathrm{ad}}$ value of 0.1891 , and was used as a control protein in the characterization experiments.

AfCalAp was predicted as a cell wall or extracellular protein by the PSORT II program. The ORF of AfCalA is $534 \mathrm{bp}$, coding for a protein of 177 amino acids with an approximate molecular mass of $18.7 \mathrm{kDa}$ and $\mathrm{pI}$ of 4.2 (as estimated using the EDITSEQ program of DNASTAR). SignalP 3.0 identified a signal peptide spanning amino acids 1-18 (Supplementary Fig. S1 available with the online journal). The thaumatin consensus was found to be localized between amino acids 73 and165 of AfCalAp by InterProScan.

Recent observations show that several of the plant thaumatin-like proteins bind and hydrolyse $1,3-\beta$-glucans of the type commonly found in fungal cell walls (Osmond et al., 2001; Trudel et al., 1998). Thaumatin-like proteins from several basidiomycete fungi exhibited 1,3- $\beta$-glucanase activity (Osmond et al., 2001; Trudel et al., 1998). Their biological role in fungi is under investigation. To compare its sequence with previously known thaumatin consensus containing proteins, AfCalAp was aligned with PR-5d, an antifungal thaumatin domain protein from Nicotiana tobacum [identity $=18.0 \%$, positives (amino acids replaced by amino acids of a similar nature $)=15.3 \%$ ] and sweet tasting thaumatin domain protein, thaumatin A, from Thaumatococcus daniellii (identity $=17.6 \%$, positives $=$ $14.0 \%)$. No significant homology between AfCalAp and these proteins could be detected and amino acid residues crucial for imparting antifungal activity or sweet taste to different thaumatin domain proteins were found to be absent (Koiwa et al., 1999) (Supplementary Fig. S1 available with the online journal). Disruption of $\operatorname{Cet} A$ and CalA (CetA/CalA double mutant), AfCalA homologues 
Table 1. Proteins assigned with maximum $P_{\text {ad }}$ values in the proteome of $A$. fumigatus

\begin{tabular}{|lll|}
\hline Gene ID & \multicolumn{1}{c|}{ Protein name } & $\boldsymbol{P}_{\text {ad }}$ value \\
\hline Afu3g00880 & Conserved hypothetical protein & 0.975974 \\
Afu6g13260 & RanBP1 domain protein & 0.973989 \\
Afu4g03240 & Cell wall galactomannoprotein Mp1 & 0.972813 \\
Afu4g11070 & Nucleoporin (SONB), putative & 0.972522 \\
Afu6g03230 & Cell wall glucanase, putative & 0.971113 \\
Afu1g16190 & Cell wall glucanase Crf1 & 0.967422 \\
Afu8g04860 & GPI-anchored glycoprotein, putative & 0.96618 \\
Afu1g05790 & GPI-anchored protein, putative & 0.960089 \\
Afu7g05340 & Conserved hypothetical protein & 0.957885 \\
Afu3g00270 & Cell wall glucanase, putative & 0.952592 \\
Afu1g14430 & Chitin-binding protein, putative & 0.950964 \\
Afu8g01520 & Pectinesterase family & 0.94821 \\
Afu6g02800 & GPI-anchored protein, putative & 0.947503 \\
Afu6g13720 & Class V chitinase, putative & 0.947318 \\
Afu1g09510 & GPI-anchored protein, putative & 0.946877 \\
Afu3g13110 & Hypothetical protein & 0.946264 \\
Afu6g12210 & Endo-1,4- $\beta$-xylanase (XynG1), putative & 0.943905 \\
Afu3g01150 & GPI-anchored cell wall protein, putative & 0.943847 \\
Afu5g09960 & GPI-anchored protein, putative & 0.943155 \\
Afu3g09690 & Extracellular thaumatin domain protein, putative & 0.942171 \\
Afu7g02460 & Hypothetical protein & 0.940627 \\
Afu3g00320 & Endo-1,4- $\beta$-xylanase (XlnA), putative & 0.938836 \\
Afu3g00420 & Acetyl xylan esterase (Axe1), putative & 0.938684 \\
Afu7g00580 & Conserved hypothetical protein & 0.936656 \\
Afu8g01970 & Extracellular endo-polygalacturonase, putative & 0.93601 \\
\hline
\end{tabular}

in Aspergillus nidulans (E-values for protein-2 BLAST with AfCalAp are $3 \mathrm{e}^{-60}$ and $6 \mathrm{e}^{-30}$, respectively), showed a synthetic lethal phenotype, with most of its conidia completely inhibited in germination (Belaish et al., 2008).

\section{AfCalA transcripts detected in $1 \mathrm{~h}$ swollen A. fumigatus conidia}

We reasoned that adhesion-related genes might be upregulated in the presence of host ECM. To test this hypothesis, we examined the relative expression of AfCalA in $1 \mathrm{~h}$ swollen conidia in the presence and absence of A549-derived ECM. RT-PCR of AfCalA and Actin was carried out using RNA isolated from resting A. fumigatus conidia and conidia incubated for $1 \mathrm{~h}$ with RPMI 1640 medium in the presence and absence of A549 cell linederived ECM. In contrast to Actin, expression of AfCalA was not detected in resting conidia (data not shown). In conidia incubated for $1 \mathrm{~h}$ with RPMI 1640 medium, AfCalA and Actin did not show any variation in transcript levels in the presence or absence of A549-derived ECM (Supplementary Fig. S2 available with the online journal). The identity of the AfCalA and Actin transcripts was confirmed by agarose gel electrophoresis in parallel with DNA ladders and by sequencing the PCR products.

Greenstein et al. (2006) have also made similar observations for AfCalA and AnCalA, wherein transcripts for both the genes were detectable exclusively in early germinating conidia ( 1 and $2 \mathrm{~h}$ swollen conidia). In Candida albicans, expression of adhesins has been found to be modulated under different host and model conditions (Green et al., 2004; Cheng et al., 2005). However, no significant modulation could be detected in the transcript expression of AfCalA in response to exposure of conidia to A549derived ECM in comparison with the control. The conidial transcriptome, however, has been found to undergo modulation under these conditions (S. K. Upadhyay, Y. Singh, S. F. Basir \& T. Madan unpublished). AfCalA expression seems to be linked to the germination process and may not be dependent on the presence of host ECM.

\section{Anti-AfCalAp antibodies significantly bound to A. fumigatus conidia}

The binding of anti-AfCalAp serum to $2 \mathrm{~h}$ swollen conidia was found to be significantly greater $(P<0.05)$ in comparison to control serum (Fig. 2). Anti-AfCalAp serum also showed significant binding to resting conidia but it was lesser than the binding of anti-AfCalAp serum to $2 \mathrm{~h}$ swollen conidia (Fig. 2).

We examined the binding of anti-AfCalAp to the surface of resting and swollen conidia by immunofluorescence confocal microscopy (Fig. 3a, b, c, d, e, f). Serial sections and three-dimensional reconstructions of resting and $2 \mathrm{~h}$ swollen conidia (Fig. 3b, c) probed with anti-AfCalAp, also revealed the presence of AfCalAp on the conidial surface. 


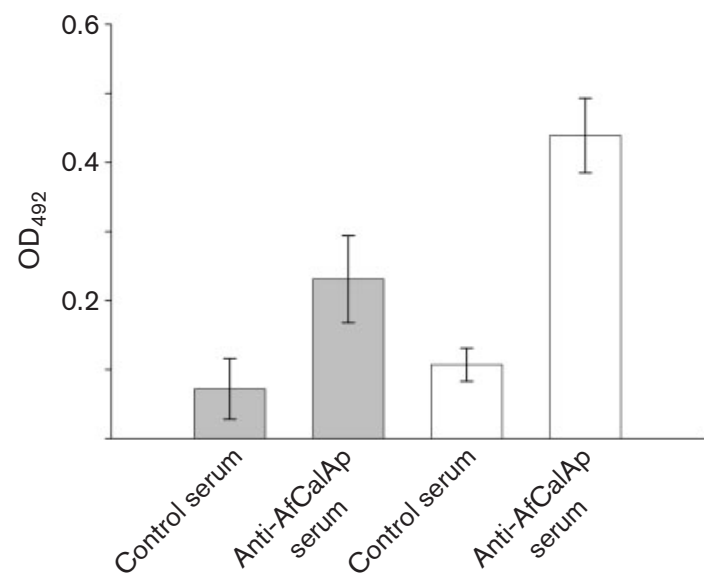

Fig. 2. Binding of anti-AfCalAp serum to $A$. fumigatus conidia adhered to a 96 -well culture plate. Data are represented as means of the $A_{492}$ of triplicate experiments \pm SD. Grey bars, serum binding with resting conidia; white bars, serum binding with $2 \mathrm{~h}$ swollen conidia.
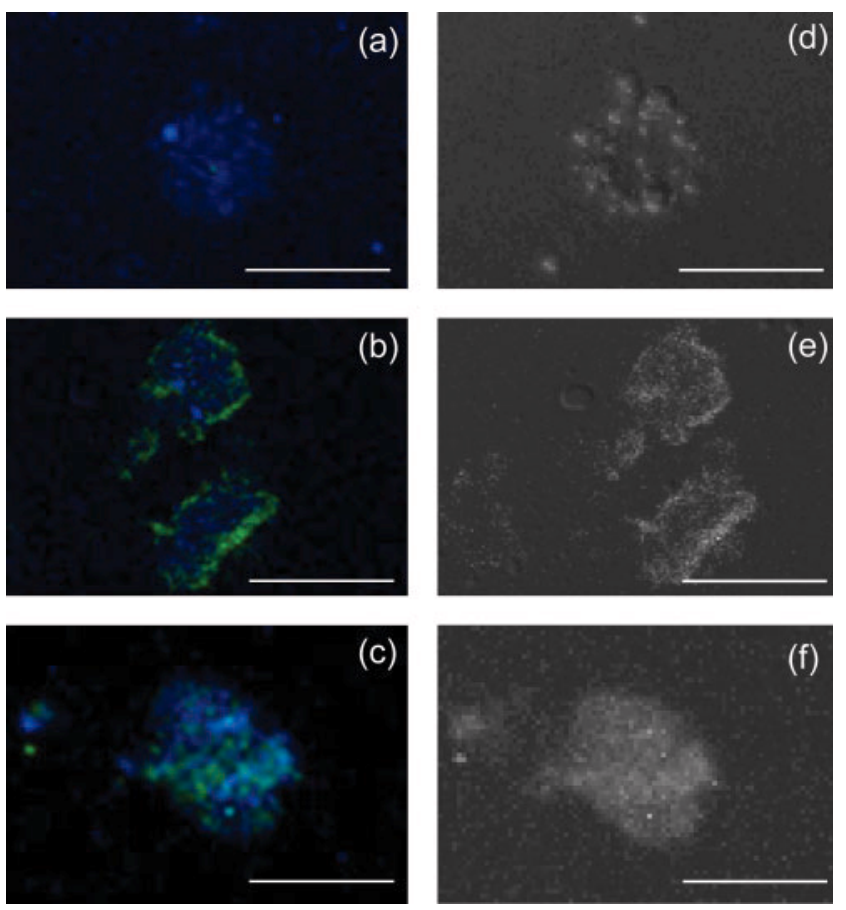

Fig. 3. Binding of anti-AfCalA serum to $A$. fumigatus conidia as revealed by immunofluorescence confocal microscopy. Conidia were incubated with mouse anti-AfCalA antibody and phycoerythrin-labelled goat anti-mouse immunoglobulins, followed by counterstaining with DAPI. Three-dimensional views were obtained after $Z$ stack by serial sections at $0.5 \mathrm{~mm}$ intervals. (a) Control sera treated conidia; (b) anti-AfCalA antibody treated conidia; (c) antiAfCalA antibody treated $2 \mathrm{~h}$ swollen conidia. (d), (e) and (f) show the phase contrast outlines of the conidia in the fluorescent images in (a), (b) and (c), respectively. Bars, $3 \mu \mathrm{m}$.
Gil et al. (1996) have already shown the binding of laminin to the surface of resting and swollen conidia using fluorescence confocal microscopy. Proteinaceous molecule(s) on the surface of conidia has/have been suggested to be responsible for their laminin affinity and a $37 \mathrm{kDa}$ protein in the conidial extract has been shown to bind to laminin. No laminin binding was observed on the hyphal surface. We have not examined the reactivity of antiAfCalAp with hyphae of A. fumigatus; however, Greenstein et al. (2006) have described the absence of AfCalA transcripts in the hyphae.

As the conditions used for interaction did not allow permeabilization of the conidia by anti-AfCalAp, the above data suggest the presence of AfCalAp on the surface of conidia. AfCalAp showed high homology and a similar expression pattern to AnCalA, its homologue (E-value $=6 \mathrm{e}^{-30}$, identity $=42 \%$, positives $=56 \%$ ) in A. nidulans (Greenstein et al., 2006), which has been shown to be localized on cell wall in geminating $(8 \mathrm{~h})$ conidia (Belaish et al., 2008).

Similar to AnCalA, AfCalAp possesses an 18-residue Nterminal signal sequence (as identified using SignalP 3.0). The presence of the signal peptide suggests AfCalAp is a secreted protein. Furthermore, we found reactivity of the anti-AfCalAp serum with a third week culture filtrate of $A$. fumigatus (data not shown).AfCalAp on the cell wall might be predicted to be present only during transit; however, this does not abrogate the adhesin characteristics of AfCalAp, as to the function as an adhesin, the protein need not necessarily be permanently present on the cell surface (Klein, 2000). The WI-1 adhesin of Blastomyces dermatitidis is secreted in the extracellular space and is present on the cell wall as well. It has been proposed that this WI-1 adhesin achieves a cell wall localization postsecretion by forming disulfide bridges with covalently attached cell wall proteins and by binding non-covalently to chitin fibrils (Brandhorst \& Klein, 2000). The presence of multiple cysteine residues in AfCalAp may facilitate a disulfide-bond-based anchorage of AfCalAp on the $A$. fumigatus cell wall, as proposed for WI-1 adhesin. Noncovalent interactions may also play a role in the cell wall localization of AfCalAp, as numerous thaumatin domain proteins have been shown to bind $\beta$-glucan in the fungal cell walls (Osmond et al., 2001; Trudel et al., 1998).

A significantly higher immunoreactivity of the $2 \mathrm{~h}$ swollen A. fumigatus conidia than the resting conidia with antiAfCalAp serum was revealed by ELISA, corroborates our RT-PCR results, wherein AfCalA transcripts were detected only in the swollen conidia and were absent in the resting conidia. The increased immunoreactivity of swollen conidia towards anti-AfCalAp serum thus seems to be an outcome of increased expression of AfCalA during the early stage of germination. However, immunofluorescent staining does not clearly show a higher level of staining in the $2 \mathrm{~h}$ swollen conidia. This may be because the swollen conidia are larger and the binding is distributed less densely. 
AfCalAp has sequence homology with a putative glycosylphosphatidylinositol (GPI)-anchored cell wall protein (Afu3g00510, E-value $=4 \mathrm{e}^{-36}$ ), a Bys1 family protein (Afu2g04533, E-value $=5 \mathrm{e}^{-04}$ ) and BYS1 domain protein (Afu5g01990, E-value $=1 \mathrm{e}^{-07}$ ) of A. fumigatus, with predicted cell wall (or extracellular, as predicted using PSORT II) localization (data not shown). It is plausible that the presence of AfCalAp homologues on the conidial surface may also contribute to the anti-AfCalAp serum reactivity of resting A. fumigatus conidia. However, the presence of AfCalAp on resting conidia, as a carried over translation product from their parent conidiating hyphae, cannot be ruled out and needs further investigation.

\section{AfCalAp interaction with laminin}

Dot-blots showed significant binding of AfCalAp with laminin in comparison with the control protein (BSA) or other ECM components (Fig. 4a). ELISA results with laminin-coated plates were in concordance with the dotblot results (Fig. 4b). Furthermore, as revealed by an ELISA-based inhibition assay, laminin binding of AfCalAp was inhibited in a dose-dependent manner in the presence of anti-AfCalAp serum (Table 2).

Laminin receptors have been detected as being distributed uniformly on the surface of $A$. fumigatus conidia and a $72 \mathrm{kDa}$ protein was identified as reacting specifically with laminin (Tronchin et al., 1997). Thus, it is plausible that

(a)

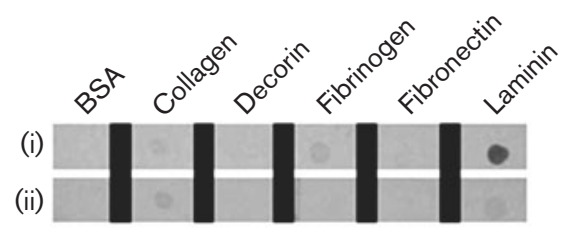

(b)

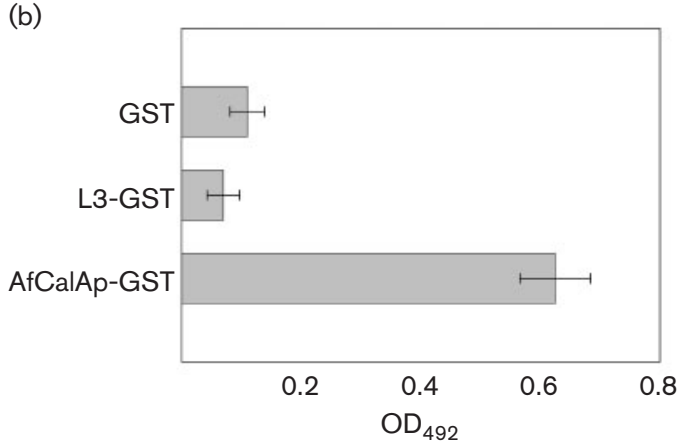

Fig. 4. AfCalAp binding to laminin. (a) Binding of AfCalAp to various ECM components studied by dot-blot: (i) binding of AfCalAp-GST to ECM components or control protein (BSA); (ii) binding of GST to ECM components or control protein. (b) Laminin binding of AfCalAp examined using ELISA (L3 is a ribosomal protein of $A$. fumigatus with a $P_{\text {ad }}$ value $\left.=0.1891\right)$. Each experiment was repeated three times with similar results.
Table 2. Inhibition of AfCalAp-GST binding to laminin by the presence of various dilutions of anti-AfCalAp serum

Each experiment was repeated three times and each $A_{492}$ value represents the mean of triplicate data points obtained at one time.

\begin{tabular}{|llllll|}
\hline \multirow{2}{*}{$\begin{array}{l}\text { Serum } \\
\text { dilution }\end{array}$} & \multicolumn{2}{c}{$\begin{array}{c}\text { Anti-AfCalAp } \\
\text { serum present }\end{array}$} & & \multicolumn{2}{c|}{$\begin{array}{c}\text { Control serum } \\
\text { present }\end{array}$} \\
\cline { 2 - 3 } \cline { 6 - 6 } & $A_{\mathbf{4 9 2}}$ & SD & & $A_{492}$ & SD \\
\hline $1: 1000$ & 0.5367 & 0.012 & & 0.5883 & 0.0158 \\
$1: 500$ & 0.4312 & 0.0208 & & 0.4702 & 0.0305 \\
$1: 200$ & 0.312 & 0.0145 & & 0.45 & 0.0115 \\
$1: 100$ & 0.25 & 0.0145 & & 0.4133 & 0.012 \\
$1: 50$ & 0.192 & 0.01 & & 0.39 & 0.0306 \\
& & & & & \\
\hline
\end{tabular}

along with the $72 \mathrm{kDa}$ laminin-binding protein, AfCalAp also contributes to the laminin binding of A. fumigatus conidia.

\section{AfCalAp binding to murine lung and spleen cells}

Flow-cytometry data showed significantly higher affinity of murine lung cells towards AfCalAp in comparison with the control protein (Fig. 5a, b, c, d). Furthermore, AfCalAp showed higher binding with the lung cells in comparison to spleen cells (Fig. 5d).

The binding of recombinant AfCalAp to the murine lung and spleen cells suggests its relevance in the adhesion of fungal conidia to host cells. Earlier studies have shown the binding of A. fumigatus conidia to pulmonary epithelial cells. Lung epithelial cells have been shown to express receptors in response to stimulation by gamma interferon, leading to their increased avidity to A. fumigatus conidia (Bromley \& Donaldson, 1996). Variable expression of such receptor molecules on lung/spleen cells could explain the higher affinity of lung cell suspension than splenic cell suspension. These receptor molecules might be able to bind AfCalAp directly or through the affinity held laminin molecules. It would be worthwhile to identify the AfCalAp receptors on host cells.

\section{Detection of IgG and IgE antibodies to AfCalAp in the sera of ABPA patients}

The immunoreactivity of AfCalAp was examined using serum from seven ABPA patients and seven healthy controls. Serum from four of the ABPA patients showed IgG reactivity towards AfCalAp, whereas only two of the ABPA patients showed IgE reactivity towards AfCalAp. None of the healthy controls showed the presence of IgG or IgE antibodies to AfCalAp (Fig. 6). These results were further confirmed by ELISA (data not shown).

The host generates antibodies to pathogenic proteins. Hence, we examined AfCalAp-specific IgG and IgE antibodies in the sera of ABPA patients. The presence of 
(a)

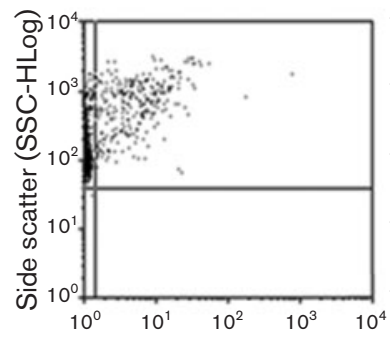

(b)

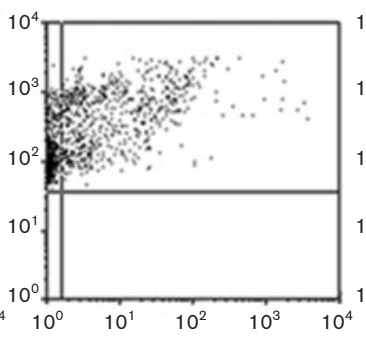

Green fluorescence (GRN-HLog)

(d)

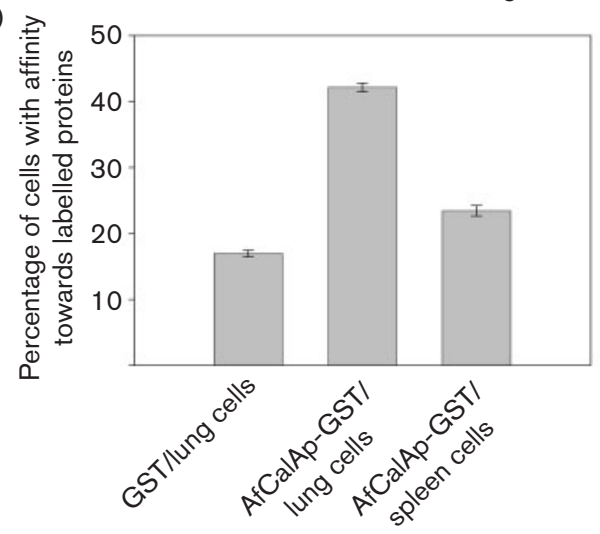

(c)

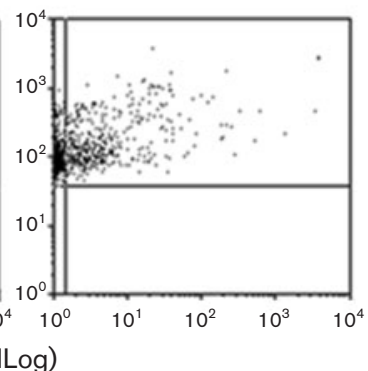

Fig. 5. Binding of AfCalAp-GST and GST (equal moles of each protein were used) with murine lung and spleen cell suspension examined by flow-cytometry using FITClabelled AfCalAp-GST and GST (number of cells counted for each sample $=5000$ ). Dots in the upper right quadrant represent the fraction of cells with binding affinity towards the labelled proteins. (a) Binding of GST with lung cells; (b) binding of AfCalAp-GST with lung cells; (c) binding of AfCalAp-GST with spleen cells; (d) histogram showing the percentage of cells in the right quadrants of (a), (b) and (c). Each experiment was repeated three times and the values represent the means of triplicate sets from three different experiments. specific IgG and IgE antibodies in four and two out of seven patients, respectively, suggests extracellular thaumatin domain protein as a minor allergen/antigen of $A$. fumigatus. Allergenicity of Mal d 2, a thaumatin-like protein of apple (Malus domestica), has been reported (Krebitz et al., 2003).

(a)

\begin{tabular}{|c|c|c|c|c|c|c|c|}
\hline & $\mathrm{P} 1$ & $\mathrm{P} 2$ & P3 & $\mathrm{P} 4$ & P5 & P6 & $\mathrm{P} 7$ \\
\hline ABPA serum & $\checkmark$ & - & $\bullet$ & $\bullet$ & & & \\
\hline
\end{tabular}

Control serum

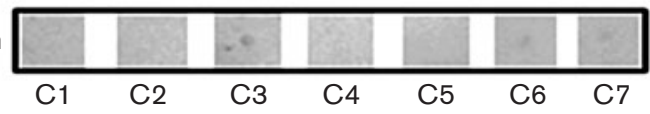

(b)

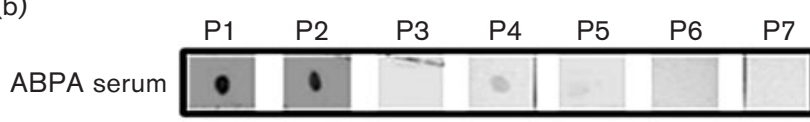

Control serum

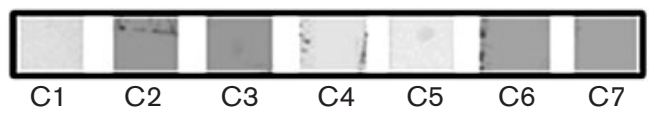

Fig. 6. Immunoreactivity of AfCalAp determined by dot-blot using diluted serum from ABPA patients (P1-P7) and healthy controls (C1-C7). (a) lgG reactivity of ABPA patients and healthy controls examined using 1:100 diluted serum, (b) lgE reactivity of ABPA patients and healthy controls examined using $1: 20$ diluted serum. The experiment was repeated three times with the same results.
Antibodies against adhesins have been shown to be effective in the inhibition of microbial adhesion to host surfaces and have been proposed as an effective therapeutic strategy (Chaturvedi et al., 2005; Masuoka et al., 1999). Anti-AfCalAp significantly inhibited the interaction of laminin with AfCalAp, suggesting that specific AfCalAp antibodies could be useful for the prevention and therapy of aspergillosis.

\section{ACKNOWLEDGEMENTS}

This investigation was financially supported by the Council of Scientific and Industrial Research, New Delhi, India. We thank Dr S. Ramachandran (Institute of Genomics and Integrative Biology, Delhi) for his help in screening of adhesins of $A$. fumigatus using SPAAN and Professor A. Shah of Vallabhbhai Patel Chest Institute, Delhi, India, for providing patient samples.

\section{REFERENCES}

Annaix, V., Bouchara, J. P., Larcher, G., Chabasse, D. \& Tronchin, G. (1992). Specific binding of human fibrinogen fragment $D$ to Aspergillus fumigatus conidia. Infect Immun 60, 1747-1755.

Belaish, R., Sharon, H., Levdansky, E., Greenstein, S., Shadkchan, Y. \& Osherov, N. (2008). The Aspergillus nidulans cetA and calA genes are involved in conidial germination and cell wall morphogenesis. Fungal Genet Biol 45, 232-242.

Bodey, G. P. \& Vartivarian, S. (1989). Aspergillosis. Eur J Clin Microbiol Infect Dis 8, 413-437.

Bouchara, J. P., Sanchez, M., Chevailler, A., Marot-Leblond, A., Lissitzky, J. C., Tronchin, G. \& Chabasse, D. (1997). Sialic acid- 
dependent recognition of laminin and fibrinogen by Aspergillus fumigatus conidia. Infect Immun 65, 2717-2724.

Bradford, M. M. (1976). A rapid and sensitive method for the quantitation of microgram quantities of protein utilizing the principle of protein-dye binding. Anal Biochem 72, 248-254.

Brandhorst, T. \& Klein, B. (2000). Cell wall biogenesis of Blastomyces dermatitidis. Evidence for a novel mechanism of cell surface localization of a virulence-associated adhesin via extracellular release and reassociation with cell wall chitin. J Biol Chem 275, 7925-7934.

Bromley, I. M. \& Donaldson, K. (1996). Binding of Aspergillus fumigatus spores to lung epithelial cells and basement membrane proteins, relevance to the asthmatic lung. Thorax 51, 1203-1209.

Chaturvedi, A. K., Kavishwar, A., Shiva Keshava, G. B. \& Shukla, P. K. (2005). Monoclonal immunoglobulin G1 directed against Aspergillus fumigatus cell wall glycoprotein protects against experimental murine aspergillosis. Clin Diagn Lab Immunol 12, 1063-1068.

Cheng, G., Wozniak, K., Walling, M. A., Fidel, P. L., Jr, Trupin, S. R. \& Hoyer, L. L. (2005). Comparison between Candida albicans agglutinin-like sequence gene expression patterns in human clinical specimens and models of vaginal candidiasis. Infect Immun 73, 1656-1663.

Coulot, P., Bouchara, J. P., Renier, G., Annaix, V., Planchenault, C., Tronchin, G. \& Chabasse, D. (1994). Specific interaction of Aspergillus fumigatus with fibrinogen and its role in cell adhesion. Infect Immun 62, 2169-2177.

Gaur, N. K. \& Klotz, S. A. (1997). Expression, cloning, and characterization of a Candida albicans gene, ALA1, that confers adherence properties upon Saccharomyces cerevisiae for extracellular matrix proteins. Infect Immun 65, 5289-5294.

Gil, M. L., Penalver, M. C., Lopez-Ribot, J. L., O’Connor, J. E. \& Martinez, J. P. (1996). Binding of extracellular matrix proteins to Aspergillus fumigatus conidia. Infect Immun 64, 5239-5247.

Gonzalez, A., Gomez, B. L., Restrepo, A., Hamilton, A. J. \& Cano, L. E. (2005). Recognition of extracellular matrix proteins by Paracoccidioides brasiliensis yeast cells. Med Mycol 43, 637-645.

Green, C. B., Cheng, G., Chandra, J., Mukherjee, P., Ghannoum, M. A. \& Hoyer, L. L. (2004). RT-PCR detection of Candida albicans ALS gene expression in the reconstituted human epithelium (RHE) model of oral candidiasis and in model biofilms. Microbiology 150, 267-275.

Greenstein, S., Shadkchan, Y., Jadoun, J., Sharon, C., Markovich, S. \& Osherov, N. (2006). Analysis of the Aspergillus nidulans thaumatinlike $\operatorname{cet} A$ gene and evidence for transcriptional repression of pyr4 expression in the cetA-disrupted strain. Fungal Genet Biol 43, 42-53.

Holmes, K. \& Fowlkes, B. J. (1996). Immunofluorescence and cell sorting. In Current Protocols in Immunology, 2nd edn. Edited by J. E. Coligan, A. M. Kruisbeek, D. H. Margulies, E. M. Shevach \& W. Strober. New York: Wiley.

Klein, B. S. (2000). Molecular basis of pathogenicity in Blastomyces dermatitidis: the importance of adhesion. Curr Opin Microbiol 3, 339-343.

Koiwa, H., Kato, H., Nakatsu, T., Oda, J., Yamada, Y. \& Sato, F. (1999). Crystal structure of tobacco PR-5d protein at $1.8 \AA$ resolution reveals a conserved acidic cleft structure in antifungal thaumatin-like proteins. J Mol Biol 286, 1137-1145.
Krebitz, M., Wagner, B., Ferreira, F., Peterbauer, C., Campillo, N., Witty, M., Kolarich, D., Steinkellner, H., Scheiner, O. \& Breiteneder, H. (2003). Plant-based heterologous expression of Mal d 2, a thaumatinlike protein and allergen of apple (Malus domestica), and its characterization as an antifungal protein. J Mol Biol 329, 721-730.

Levdansky, E., Romano, J., Shadkchan, Y., Sharon, H., Verstrepen, K. J., Fink, G. R. \& Osherov, N. (2007). Coding tandem repeats generate diversity in Aspergillus fumigatus genes. Eukaryot Cell 6, $1380-1391$.

Lopez-Ribot, J. L. \& Chaffin, W. L. (1994). Binding of the extracellular matrix component entactin to Candida albicans. Infect Immun 62, $4564-4571$.

Masuoka, J., Wu, G., Glee, P. M. \& Hazen, K. C. (1999). Inhibition of Candida albicans attachment to extracellular matrix by antibodies which recognize hydrophobic cell wall proteins. FEMS Immunol Med Microbiol 24, 421-429.

Mendes-Giannini, M. J., Andreotti, P. F., Vincenzi, L. R., da Silva, J. L., Lenzi, H. L., Benard, G., Zancope-Oliveira, R., de Matos Guedes, H. L. \& Soares, C. P. (2006). Binding of extracellular matrix proteins to Paracoccidioides brasiliensis. Microbes Infect 8, 1550-1559.

Osmond, R. I., Hrmova, M., Fontaine, F., Imberty, A. \& Fincher, G. B. (2001). Binding interactions between barley thaumatin-like proteins and $(1,3)-\beta$-D-glucans. Kinetics, specificity, structural analysis and biological implications. Eur J Biochem 268, 4190-4199.

Penalver, M. C., O’Connor, J. E., Martinez, J. P. \& Gil, M. L. (1996). Binding of human fibronectin to Aspergillus fumigatus conidia. Infect Immun 64, 1146-1153.

Rosenberg, M., Patterson, R., Mintzer, R., Cooper, B. J., Roberts, M. \& Harris, K. E. (1977). Clinical and immunologic criteria for the diagnosis of allergic bronchopulmonary aspergillosis. Ann Intern Med 86, 405-414.

Sachdeva, G., Kumar, K., Jain, P. \& Ramachandran, S. (2005). SPAAN: a software program for prediction of adhesins and adhesinlike proteins using neural networks. Bioinformatics 21, 483-491.

Saxena, S., Madan, T., Muralidhar, K. \& Sarma, P. U. (2003). cDNA cloning, expression and characterization of an allergenic L3 ribosomal protein of Aspergillus fumigatus. Clin Exp Immunol 134, 86-91.

Sturtevant, J. E. \& Latge, J. P. (1992). Interactions between conidia of Aspergillus fumigatus and human complement component C3. Infect Immun 60, 1913-1918.

Thau, N., Monod, M., Crestani, B., Rolland, C., Tronchin, G., Latge, J. P. \& Paris, S. (1994). rodletless mutants of Aspergillus fumigatus. Infect Immun 62, 4380-4388.

Tronchin, G., Esnault, K., Renier, G., Filmon, R., Chabasse, D. \& Bouchara, J. P. (1997). Expression and identification of a lamininbinding protein in Aspergillus fumigatus conidia. Infect Immun 65, 9-15.

Trudel, J., Grenier, J., Potvin, C. \& Asselin, A. (1998). Several thaumatin-like proteins bind to $\beta$-1,3-glucans. Plant Physiol 118, 1431-1438.

Wasylnka, J. A. \& Moore, M. M. (2000). Adhesion of Aspergillus species to extracellular matrix proteins, evidence for involvement of negatively charged carbohydrates on the conidial surface. Infect Immun 68, 3377-3384. 